\title{
Duration-response association between exercise and HDL in both male and female Taiwanese adults aged 40 years and above
}

\author{
Cheng-Feng Jan', Hui-Chin Chang ${ }^{2,3}$, Disline Manli Tantoh ${ }^{2}$, Pei-Hsin Chen ${ }^{2}$, Wen- \\ Hsiu Liu ${ }^{2}$, Jing-Yang Huang ${ }^{2}$, Min-Chen $W_{u^{1}}$ and Yung-Po Liaw ${ }^{2,4}$ \\ ${ }^{1}$ Office of Physical Education, Chung Yuan Christian University, Taoyuan City, Taiwan \\ ${ }^{2}$ Department of Public Health and Institute of Public Health, Chung Shan Medical University, Taichung City, Taiwan \\ ${ }^{3}$ Library, Chung Shan Medical University Hospital, Taichung City, Taiwan \\ ${ }^{4}$ Department of Family and Community Medicine, Chung Shan Medical University Hospital, Taichung City, Taiwan \\ Correspondence to: Yung-Po Liaw, email: Liawyp@csmu.edu.tw \\ Keywords: HDL; exercise; duration-response; Health Promotion Administration; Taiwan \\ Received: June 10,2017 Accepted: December 01,2017～Published: December 14, 2017 \\ Copyright: Jan et al. This is an open-access article distributed under the terms of the Creative Commons Attribution License 3.0 \\ (CC BY 3.0), which permits unrestricted use, distribution, and reproduction in any medium, provided the original author and source \\ are credited.
}

\section{ABSTRACT}

Background: Exercise is an important cardiovascular risk reducing therapy.

Objective: The aim of this study was to assess the relationship between weekly exercise duration and high-density lipoprotein cholesterol (HDL-c) in Taiwanese men and women.

Methods: Data were retrieved from the dataset of the national adult preventive medical services which is recorded under the Health Promotion Administration (HPA). The lipid profiles of 194528 eligible participants aged 40 years and above who completed a questionnaire on recent health behavior including smoking, drinking, exercise and other factors in 2014 were determined. Weekly exercise durations of 0.0 , $<2.5$ and $\geq 2.5$ hours were classified as no, below recommended and recommended, respectively. The relationship between exercise and HDL-c was determined using linear regression.

Results: After multivariate adjustments, a duration-response association existed between exercise and HDL-C $(P$-trend $<0.0001)$ in both sexes. Weekly exercise durations of $<2.5$ and $\geq 2.5$ hours were both positively associated with HDL-C (P $<0.0001)$ in both sexes. However, the associations were stronger in males than females in both exercise groups. Smoking $(P<0.05)$ and BMI $(P<0.0001)$ were negatively associated while drinking was positively associated with HDL-c in both sexes.

Conclusion: This study demonstrated a duration-response association between exercise and HDL-C. Exercise at durations below the minimum weekly recommendation of 2.5 hours was positively associated with HDL-C.

\section{INTRODUCTION}

High-density lipoprotein (HDL) is often referred to as 'good' cholesterol because high levels can prevent or even regress atherosclerosis and other cardiovascular diseases (CVDs). This is achieved through its ability to act as a scavenger for excess cholesterols thereby preventing their accumulation [1]. That is, HDL can remove these cholesterols from the arterial tissues and transport them to the liver for breakdown and biliary excretion. Moreover, it inhibits low-density lipoprotein (LDL) oxidation and protects against thrombosis and endothelial inflammation $[2,3]$. However, at low levels, HDL-c could increase the risk of CVDs [4-7]. CVDs are the leading cause of 
deaths and accounted for about $31 \%$ of all global deaths in 2015 [8,9]. The risk of CVDs is partly prevented and or reduced by exercise which is capable of increasing HDL-c levels in addition to reducing oxidative stress [10-12]. The duration of exercise is one of the paramount elements in the relationship between HDL and exercise. The world health organization (WHO) recommends a minimum of 2.5 hours (150 minutes) of weekly moderateintensity aerobic exercise for those aged 18-64 years as well as 65 years and above [13]. Kodama and colleagues concluded in their review that the minimum total weekly exercise duration that can significantly raise HDL-c levels is 2 hours [11]. There have been inconsistent results regarding the association between HDL-c and exercise $[1,14,15]$. Besides, few studies have been conducted to assess such an association in Taiwan. Lifestyle change is one of the factors that account for the high prevalence of hyperlipidemia in Taiwan [16]. Regular exercise is one of the first steps taken to manage lipid levels. This study was therefore aimed at assessing the relationship between exercise and HDL-c in Taiwanese men and women aged 40 years and above.

\section{RESULTS}

Tables 1 and 2 show the baseline characteristics of the male and female participants, respectively. The study participants comprised 83681 males and 110847 females. Among both male and female participants, more than $50 \%$ were sedentary. There were significant differences $(\mathrm{P}<0.0001)$ in HDL-c among various exercise groups in both males and females. Among the males, the HDL-c/mg/dl of those who did no exercise was lowest $(49.47 \pm 17.82)$ when compared to that of those who did $<2.5$ hours $(51.04 \pm 19.77)$ and $\geq 2.5$ hours $(53.32 \pm 21.44)$ of weekly exercise (Table 1). In the same way, the HDL-c/mg/dl of female participants who did no exercise was lowest $(57.79 \pm 18.64)$ when compared to that of those who did $<2.5$ hours $(59.52 \pm 20.08)$ and $\geq 2.5$ hours $(61.31 \pm 21.43)$ of weekly exercise (Table 2). However, the female participants had higher HDL-c than their male counterparts in all the exercise groups.

After adjusting for smoking, drinking, BMI, lipoproteins (total cholesterol, LDL, triglycerides), age, area (Taichung city and Yunlin county), betel nut chewing, disease history (hypertension, diabetes, hyperlipidemia, stroke, heart and kidney disease), waist circumference, systolic and diastolic blood pressures, blood glucose, creatinine, ALT and eGFR, there was a duration-response association between exercise and HDL-c ( $\mathrm{P}$ for the trend $<0.0001$ ) in both males and females (Tables 3 and 4). Weekly exercise durations of $<2.5$ and $\geq 2.5$ hours were both positively correlated with HDL-c $(\mathrm{P}<0.0001)$ in both sexes. However, these correlations were stronger in males than females in both exercise groups (Tables 3 and 4). Among the male participants, the HDL-c of those who did $<2.5$ hours of exercise per week was lower (B $=0.392$ ) compared to that of those who did $\geq 2.5$ hours per week $(\mathrm{B}=0.780)$. Among the female participants, the HDL-c of those who did $<2.5$ hours of exercise per week was lower $(\mathrm{B}=0.370)$ than that of those who did $\geq 2.5$ hours of exercise per week $(\mathrm{B}=0.395)$. HDL-c was negatively correlated with smoking $(\mathrm{P}<0.05)$ and BMI ( $\mathrm{P}$ $<.0001$ ) in both sexes (Tables 3 and 4). However, it was not significantly correlated with occasional smoking. On the other hand, drinking was positively correlated with HDL-c in both males and females (Tables 3 and 4).

\section{DISCUSSION}

To our knowledge, this study is the first to use the WHO exercise recommendation for health to investigate the duration-response association between exercise and HDL-c in Taiwanese men and women using data from the adult preventive medical services dataset.

In our study, male HDL-c at baseline was lower than that of females. This has been previously reported [17, 18]. Individuals with low HDL-c levels are predisposed to a greater risk of CVDs [4-7]. Fortunately, both males and females can increase their HDL-c levels through exercise. In this study, both exercise durations of $<2.5$ and $\geq 2.5$ hours per week were positively correlated with HDL-c in both sexes. This shows how important exercise is in preventing CVDs even for those who cannot meet the minimum recommended weekly duration. However, meeting this recommended duration is still necessary and should be encouraged. Different studies have shown varied results on the positive effect of physical activity on HDL $[11,14,15,19-22]$. Individuals with low baseline HDL-c levels have been shown to have greatest increases after exercise [21]. Sex, exercise duration, and intensity are among the factors that account for differences in lipoprotein responses to exercise [23]. In the current study, the HDL-c of males was higher than that of their female counterparts after exercise. This is consistent with a previous report [18]. Men are likely to involve in higher exercise intensity and duration than women even though our study did not investigate this. The HDL raising and cardioprotective mechanism of exercise are yet to be clearly understood. However, this can be partly explained by the enzymatic metabolism of lipoproteins especially increased lipoprotein lipase and reduced hepatic lipase activity [18, 22, 24, 25]. Moderate alcohol intake is a known HDL-c raising factor [26, 27]. In the current study, drinking was positively correlated with HDL-c. Increased transport rate of HDL apolipoprotein, increased lipoprotein lipase and decreased hepatic lipase activity have been suggested as the underlying mechanisms for this relation $[26,27]$. However, too much alcohol consumption should not be recommended for that purpose due to its deleterious effects when abused. In agreement with previous studies, smoking and BMI were negatively correlated with 
Table 1: Basic descriptive characteristics of the male participants by exercise

\begin{tabular}{|c|c|c|c|c|}
\hline \multirow{2}{*}{ Variable } & \multicolumn{3}{|c|}{ Exercise/hours/week } & \multirow{2}{*}{ P-value } \\
\hline & $0.0 \mathrm{n}=43322(51.77 \%)$ & $<2.5 \mathrm{n}=28869(34.50 \%)$ & $\geq 2.5 \mathrm{n}=11490(13.73 \%)$ & \\
\hline Age (years) & $62.85 \pm 13.34$ & $63.65 \pm 12.64$ & $65.47 \pm 12.17$ & $<0.0001$ \\
\hline Area & & & & $<0.0001$ \\
\hline Taichung & $30966(71.48 \%)$ & $23032(79.78 \%)$ & $9411(81.91 \%)$ & \\
\hline Yunlin & $12356(28.52 \%)$ & $5837(20.22 \%)$ & $2079(18.09 \%)$ & \\
\hline Smoking status & & & & $<0.0001$ \\
\hline Never & $32435(74.87 \%)$ & $22289(77.21 \%)$ & $9691(84.34 \%)$ & \\
\hline Occasional & $2135(4.93 \%)$ & $1646(5.70 \%)$ & $434(3.78 \%)$ & \\
\hline$<1 \mathrm{pack} /$ day & $5349(12.35 \%)$ & $3446(11.94 \%)$ & $999(8.69 \%)$ & \\
\hline$\geq 1 \mathrm{pack} / \mathrm{day}$ & $3403(7.86 \%)$ & $1488(5.15 \%)$ & $366(3.19 \%)$ & \\
\hline Drinking status & & & & $<0.0001$ \\
\hline Never & $32613(75.28 \%)$ & $20239(70.11 \%)$ & $8135(70.80 \%)$ & \\
\hline Occasional & $8017(18.51 \%)$ & $7296(25.27 \%)$ & $2882(25.08 \%)$ & \\
\hline Frequent & $2692(6.21 \%)$ & $1334(4.62 \%)$ & $473(4.12 \%)$ & \\
\hline Betel nut status & & & & $<0.0001$ \\
\hline Never & $39220(90.53 \%)$ & $26974(93.44 \%)$ & $11092(96.54 \%)$ & \\
\hline Occasional & $2363(5.45 \%)$ & $1240(4.30 \%)$ & $301(2.62 \%)$ & \\
\hline Frequent & $1739(4.01 \%)$ & $655(2.27 \%)$ & $97(0.84 \%)$ & \\
\hline \multicolumn{5}{|l|}{ Disease history } \\
\hline Hypertension & $12458(28.76 \%)$ & $10007(34.66 \%)$ & $4368(38.02 \%)$ & $<0.0001$ \\
\hline Diabetes & $5812(13.42 \%)$ & $4205(14.57 \%)$ & $1781(15.50 \%)$ & $<0.0001$ \\
\hline Hyperlipidemia & $2780(6.42 \%)$ & $2015(6.98 \%)$ & $1212(10.55 \%)$ & $<0.0001$ \\
\hline Heart disease & $2830(6.53 \%)$ & $2159(7.48 \%)$ & $1028(8.95 \%)$ & $<0.0001$ \\
\hline Stroke & $1195(2.76 \%)$ & $564(1.95 \%)$ & $207(1.80 \%)$ & $<0.0001$ \\
\hline Kidney disease & $1003(2.32 \%)$ & $505(1.75 \%)$ & $238(2.07 \%)$ & $<0.0001$ \\
\hline Height $(\mathrm{cm})$ & $165.63 \pm 6.64$ & $165.79 \pm 6.51$ & $165.91 \pm 6.42$ & $<0.0001$ \\
\hline Weight (kg) & $68.64 \pm 11.85$ & $68.8 \pm 10.96$ & $68.37 \pm 10.48$ & 0.0029 \\
\hline $\mathrm{BMI}\left(\mathrm{kg} / \mathrm{m}^{2}\right)$ & $24.97 \pm 3.73$ & $24.98 \pm 3.42$ & $24.80 \pm 3.29$ & $<0.0001$ \\
\hline Waist circumference $(\mathrm{cm})$ & $71.29 \pm 26.50$ & $72.50 \pm 25.41$ & $74.00 \pm 24.38$ & $<0.0001$ \\
\hline SBP (mmHg) & $132.11 \pm 18.48$ & $132.69 \pm 18.29$ & $132.63 \pm 18.16$ & $<0.0001$ \\
\hline $\mathrm{DBP}(\mathrm{mmHg})$ & $79.71 \pm 11.87$ & $79.47 \pm 11.53$ & $79.22 \pm 11.14$ & $<0.0001$ \\
\hline Total cholesterol (mg/dl) & $187.08 \pm 39.28$ & $188.14 \pm 37.10$ & $187.40 \pm 36.07$ & 0.0011 \\
\hline LDL-c (mg/dl) & $109.67 \pm 36.30$ & $110.32 \pm 35.72$ & $108.91 \pm 35.38$ & 0.0011 \\
\hline HDL-c (mg/dl) & $49.47 \pm 17.82$ & $51.04 \pm 19.77$ & $53.32 \pm 21.44$ & $<0.0001$ \\
\hline Triglyceride (mg/dl) & $144.37 \pm 90.27$ & $137.90 \pm 84.96$ & $128.08 \pm 75.21$ & $<0.0001$ \\
\hline ALT & & & & $<0.0001$ \\
\hline$\leq 40 \mathrm{IU} / 1$ & $36081(83.29 \%)$ & $24684(85.50 \%)$ & $9982(86.88 \%)$ & \\
\hline$>40 \mathrm{IU} / 1$ & $7241(16.71 \%)$ & $4185(14.50 \%)$ & $1508(13.12 \%)$ & \\
\hline Blood glucose (mg/dl) & $112.95 \pm 47.76$ & $110.99 \pm 41.27$ & $108.23 \pm 35.4$ & $<0.0001$ \\
\hline Blood creatinine (mg/dl) & $1.12 \pm 0.74$ & $1.07 \pm 0.61$ & $1.07 \pm 0.53$ & $<0.0001$ \\
\hline $\operatorname{EGFR}\left(\mathrm{ml} / \mathrm{min} / 1.73 \mathrm{~m}^{2}\right)$ & $75.71 \pm 21.87$ & $74.84 \pm 19.40$ & $73.37 \pm 18.12$ & $<0.0001$ \\
\hline
\end{tabular}


Table 2: Basic descriptive characteristics of the female study participants by exercise

\begin{tabular}{|c|c|c|c|c|}
\hline \multirow{2}{*}{ Variable } & \multicolumn{3}{|c|}{ Exercise/hours/week } & \multirow[b]{2}{*}{ P-value } \\
\hline & $0.0 n=60893(54.93 \%)$ & $<2.5 \mathrm{n}=37059(33.43 \%)$ & $\geq 2.5 \mathrm{n}=12895(11.63 \%)$ & \\
\hline Age (years) & $62.79 \pm 13.36$ & $62.61 \pm 12.08$ & $64.30 \pm 11.42$ & $<0.0001$ \\
\hline Area & & & & $<0.0001$ \\
\hline Taichung & $43662(71.70 \%)$ & $29629(79.95 \%)$ & $10766(83.49 \%)$ & \\
\hline Yunlin & $17231(28.30 \%)$ & $7430(20.05 \%)$ & $2129(16.51 \%)$ & \\
\hline Smoking status & & & & $<0.0001$ \\
\hline Never & $58214(95.60 \%)$ & $35785(96.56 \%)$ & $12702(98.50 \%)$ & \\
\hline Occasional & $464(0.76 \%)$ & $302(0.81 \%)$ & $79(0.61 \%)$ & \\
\hline$<1 \mathrm{pack} /$ day & $977(1.60 \%)$ & $454(1.23 \%)$ & $92(0.71 \%)$ & \\
\hline$\geq 1 \mathrm{pack} /$ day & $1238(2.03 \%)$ & $518(1.40 \%)$ & $22(0.17 \%)$ & \\
\hline Drinking status & & & & $<0.0001$ \\
\hline Never & $57397(94.26 \%)$ & $34573(93.29 \%)$ & $12140(94.15 \%)$ & \\
\hline Occasional & $2265(3.72 \%)$ & $1939(5.23 \%)$ & $706(5.47 \%)$ & \\
\hline Frequent & $1231(2.02 \%)$ & $547(1.48 \%)$ & $49(0.38 \%)$ & \\
\hline Betel nut status & & & & $<0.0001$ \\
\hline Never & $59664(97.98 \%)$ & $36520(98.55 \%)$ & $12864(99.76 \%)$ & \\
\hline Occasional & $189(0.31 \%)$ & $95(0.26 \%)$ & $19(0.15 \%)$ & \\
\hline Frequent & $1040(1.71 \%)$ & $444(1.20 \%)$ & $12(0.09 \%)$ & \\
\hline \multicolumn{5}{|l|}{ Disease history } \\
\hline Hypertension & $16701(27.43 \%)$ & $11941(32.22 \%)$ & $4464(34.62 \%)$ & $<0.0001$ \\
\hline Diabetes & $7853(12.90 \%)$ & $5193(14.01 \%)$ & $1793(13.90 \%)$ & $<0.0001$ \\
\hline Hyperlipidemia & $3906(6.41 \%)$ & $2697(7.28 \%)$ & $1389(10.77 \%)$ & $<0.0001$ \\
\hline Heart disease & $3930(6.45 \%)$ & $2610(7.04 \%)$ & $1024(7.94 \%)$ & $<0.0001$ \\
\hline Stroke & $1232(2.02 \%)$ & $679(1.83 \%)$ & $202(1.57 \%)$ & 0.0012 \\
\hline Kidney disease & $1059(1.74 \%)$ & $538(1.45 \%)$ & $203(1.57 \%)$ & 0.0023 \\
\hline Height (cm) & $154.29 \pm 6.20$ & $154.43 \pm 6.00$ & $154.35 \pm 5.88$ & 0.0038 \\
\hline Weight (kg) & $58.54 \pm 10.32$ & $58.30 \pm 9.66$ & $57.50 \pm 8.98$ & $<0.0001$ \\
\hline BMI $\left(\mathrm{kg} / \mathrm{m}^{2}\right)$ & $24.59 \pm 4.10$ & $24.45 \pm 3.84$ & $24.14 \pm 3.60$ & $<0.0001$ \\
\hline Waist circumference $(\mathrm{cm})$ & $66.47 \pm 24.90$ & $67.07 \pm 23.81$ & $67.92 \pm 22.78$ & $<0.0001$ \\
\hline SBP (mmHg) & $129.84 \pm 19.56$ & $129.93 \pm 19.68$ & $130.08 \pm 19.36$ & 0.4055 \\
\hline $\mathrm{DBP}(\mathrm{mmHg})$ & $76.73 \pm 11.67$ & $76.73 \pm 11.53$ & $76.28 \pm 11.06$ & $<0.0001$ \\
\hline Total cholesterol (mg/dl) & $197.67 \pm 39.28$ & $199.17 \pm 37.65$ & $199.39 \pm 37.29$ & $<0.0001$ \\
\hline LDL-c (mg/dl) & $114.95 \pm 36.02$ & $115.39 \pm 35.99$ & $114.03 \pm 35.37$ & 0.0010 \\
\hline HDL-c (mg/dl) & $57.79 \pm 18.64$ & $59.52 \pm 20.08$ & $61.34 \pm 21.43$ & $<0.0001$ \\
\hline Triglyceride (mg/dl) & $127.16 \pm 73.54$ & $123.68 \pm 70.80$ & $119.83 \pm 66.76$ & $<0.0001$ \\
\hline ALT & & & & $<0.0001$ \\
\hline$\leq 40 \mathrm{IU} / 1$ & $55101(90.49 \%)$ & $33783(91.16 \%)$ & $11885(92.17 \%)$ & \\
\hline$>40 \mathrm{IU} / 1$ & $5792(9.51 \%)$ & $91.16(8.84 \%)$ & $1010(7.83 \%)$ & \\
\hline Blood glucose (mg/dl) & $108.72 \pm 42.64$ & $106.71 \pm 36.90$ & $104.40 \pm 31.73$ & $<0.0001$ \\
\hline Blood creatinine (mg/dl) & $0.86 \pm 0.57$ & $0.81 \pm 0.46$ & $0.82 \pm 0.41$ & $<0.0001$ \\
\hline $\operatorname{EGFR}\left(\mathrm{ml} / \mathrm{min} / 1.73 \mathrm{~m}^{2}\right)$ & $77.06 \pm 22.85$ & $78.19 \pm 21.46$ & $76.68 \pm 19.97$ & $<0.0001$ \\
\hline
\end{tabular}


Table 3: Multiple linear regression showing the association of HDL with exercise in males

\begin{tabular}{|c|c|c|c|}
\hline Variable & Regression coefficient (B) & Standardized coefficient $(\beta)$ & P-value \\
\hline \multicolumn{4}{|c|}{ Exercise hours/week (Reference: 0) } \\
\hline$<2.5$ & 0.392 & 0.010 & $<0.0001$ \\
\hline$\geq 2.5$ & 0.780 & 0.014 & $<0.0001$ \\
\hline${ }^{*}$ Test for linear trend & & & $<0.0001$ \\
\hline \multicolumn{4}{|c|}{ Smoking status (Reference: Never) } \\
\hline Occasional & -0.106 & -0.001 & 0.4871 \\
\hline$<1 \mathrm{pack} /$ day & -0.653 & -0.011 & $<0.0001$ \\
\hline$\geq 1 \mathrm{pack} / \mathrm{day}$ & -0.863 & -0.011 & $<0.0001$ \\
\hline \multicolumn{4}{|c|}{ Drinking status (Reference: Never) } \\
\hline Occasional & 1.122 & 0.024 & $<0.0001$ \\
\hline Frequent & 2.454 & 0.029 & $<0.0001$ \\
\hline BMI $\left(\mathrm{kg} / \mathrm{m}^{2}\right)$ & -0.202 & -0.038 & $<0.0001$ \\
\hline Total cholesterol (mg/dl) & 0.743 & 1.485 & $<0.0001$ \\
\hline LDL-c (mg/dl) & -0.733 & -1.382 & $<0.0001$ \\
\hline Triglyceride (mg/dl) & -0.141 & -0.639 & $<0.0001$ \\
\hline
\end{tabular}

Multiple linear regression with exercise as exposure adjusted for smoking, drinking, BMI, lipoproteins (total cholesterol, LDL, triglycerides), age, area (Taichung city and Yunlin county), betel nut, disease history (hypertension, diabetes, hyperlipidemia, stroke, heart and kidney disease), waist circumference, systolic and diastolic blood pressures, blood glucose and creatinine, Alanine transaminase (ALT) and estimated glomerular filtration rate (eGFR).

Table 4: Multiple linear regression showing the association of HDL with exercise in females

\begin{tabular}{lccc}
\hline Variable & Regression coefficient (B) & Standardized coefficient $(\boldsymbol{\beta})$ & P-value \\
\hline Exercise hours/week (Reference: 0) & & & \\
$\quad<2.5$ & 0.370 & 0.009 & $<0.0001$ \\
$\geq 2.5$ & 0.395 & 0.006 & $<0.0001$ \\
$\quad{ }^{*}$ Test for linear trend & & $<0.0001$ \\
Smoking status (Reference: Never) & & & \\
$\quad$ Occasional & 0.259 & 0.001 & 0.3884 \\
$\quad<1$ pack/day & -0.667 & -0.004 & 0.0032 \\
$\geq 1$ pack/day & -0.834 & -0.005 & 0.0401 \\
Drinking status (Reference: Never) & & & \\
$\quad$ Occasional & 1.200 & 0.013 & $<.0001$ \\
Frequent & 1.981 & 0.013 & $<.0001$ \\
BMI (kg/m ${ }^{2}$ ) & -0.121 & -0.025 & $<.0001$ \\
Total cholesterol (mg/dl) & 0.784 & 1.549 & $<.0001$ \\
LDL-c (mg/dl) & -0.766 & -1.411 & $<.0001$ \\
Triglyceride (mg/dl) & -0.159 & -0.586 & $<.0001$ \\
\hline
\end{tabular}

Multiple linear regression with exercise as exposure adjusted for smoking, drinking, BMI, lipoproteins (total cholesterol, LDL, triglycerides), age, area (Taichung city and Yunlin county), betel nut, disease history (hypertension, diabetes, hyperlipidemia, stroke, heart and kidney disease), waist circumference, systolic and diastolic blood pressures, blood glucose and creatinine, Alanine transaminase (ALT) and estimated glomerular filtration rate (eGFR). 
HDL-c in our study [28, 29, 30]. Cigarette smoking and BMI negatively affect HDL-c by altering cholesterol acyltransferase (LCAT), reducing lipoprotein lipase and increasing hepatic lipase lipid transport activities [28, 31].

\section{Strengths and limitations}

The strengths of our study are that, it is the first study to demonstrate a duration-response association between exercise and HDL-c in Taiwanese adults aged 40 years and above using a big data source. Furthermore, the data were analyzed by gender and adjustments were made for confounders including age, drinking, smoking, disease history and many other factors. However, the study is limited in its cross sectional design whereby no causal relationship could be drawn. Moreover, to investigate the relationship between exercise and lipid profile, both the duration and intensity of exercise are important. However, our dataset did not contain information on the intensity of exercise.

\section{MATERIALS AND METHODS}

Data were retrieved from the national adult preventive medical services dataset which is recorded under the Health Promotion Administration (HPA). The Health Promotion Administration, formerly called the Bureau of Health Promotion, Department of Health is responsible for health promotion and non-communicable disease prevention work. It provides free triennial and annual services for adults aged 40-64 and $\geq 65$ years, respectively. It contains records of individuals who have used such services since 1996. However, a more valid electronic record was developed only from 2012. This study initially recruited 221269 male and female volunteers from Taichung city and Yunlin county. They completed a questionnaire on their recent health behaviors such as exercise, smoking, drinking and others in 2014. Moreover, they also provided information on their disease history based on their response (yes or no) to whether they have ever been diagnosed with hypertension, diabetes, hyperlipidemia, heart disease, stroke, and kidney disease. A total of 194528 eligible participations were included in the analysis. Those with missing exercise data $(n=1421)$ and incomplete records of other variables $(n=23257)$ were excluded from the analysis. In addition, outliers of HDL $(<10$ or $>200 \mathrm{mg} / \mathrm{dl}, \mathrm{n}=2063)$ were excluded from the analysis. This was because the cumulative percentage of $\mathrm{HDL}<10 \mathrm{mg} / \mathrm{dL}$ was about $1 \%$ while that of HDL $\leq 200 \mathrm{mg} / \mathrm{dL}$ was almost $99 \%$. The WHO recommends a minimum of 2.5 hours of moderate-intensity weekly exercise among adults aged 18 years and above as well as 65 years and above $[13,32]$. In this study, exercise durations of $0.0,<2.5$ and $\geq 2.5$ hours/week were classified as: no, below recommended and recommended. The dataset contains results of biochemical tests of participants including blood glucose, lipid profile, Alanine transaminase (ALT), creatinine and estimated glomerular filtration rate (eGFR.). Physical examinations (height, weight, waist circumference, body mass index (BMI) and blood pressure) are also available in the dataset. Multiple linear regression was used to determine the relationship between exercise and HDL-c. Confounding variables, some of which included age, smoking, drinking, BMI, betel nut chewing (a habit of consuming areca nuts wrapped in leaves of Piper betle), ALT and disease history were adjusted for. For ALT, the normal upper limit of 40 IU/L $[33,34]$ was used as the cut off value in our analysis. Confounders were selected based on previous studies $[15,26,28,29,35-38]$. This study was approved by the institutional review board of China Medical University and Hospital.

\section{CONCLUSION}

In conclusion, our study demonstrated a durationresponse association between exercise and HDL-c. Exercise at durations below the minimum weekly recommendation of 2.5 hours per week was positively associated with HDL-c levels. Because of this, adults should be continuously encouraged to engage in exercise. This might serve as a motivation to prevent sedentary lifestyle. However, further studies should take our limitations into consideration during their investigation.

\section{Author contributions}

(1) The conception and design of the study, or acquisition of data, or analysis and interpretation of data: All authors.

(2) Drafting of the article or revising it critically for important intellectual content: Cheng-Feng Jan, Disline Manli Tantoh and Yung-Po Liaw.

(3) Final approval of the version to be submitted. All authors.

\section{CONFLICTS OF INTEREST}

The authors declare that they have no conflicts of interest.

\section{FUNDING}

This work was supported by funds that were partly provided by the Ministry of Science and Technology (MOST), Taiwan (MOST 104-2420-H-040-001, 1042119-M-040-002, 105-2627-M-040-002 and 106-2627M-040-002). 


\section{REFERENCES}

1. Ahn N, Kim K. High-density lipoprotein cholesterol (HDLC) in cardiovascular disease: effect of exercise training. Integr Med Res. 2016; 5: 212.

2. Badimon L, Vilahur G. LDL-cholesterol versus HDLcholesterol in the atherosclerotic plaque: inflammatory resolution versus thrombotic chaos. Ann N Y Acad Sci. 2012; 1254: 18-32.

3. Tran-Dinh A, Diallo D, Delbosc S, Varela-Perez LM, Dang Q, Lapergue B, Burillo E, Michel J, Levoye A, MartinVentura J. HDL and endothelial protection. Br J Pharmacol. 2013; 169: 493-511.

4. Lazo-Porras M, Bernabe-Ortiz A, Málaga G, Gilman RH, Acuña-Villaorduña A, Cardenas-Montero D, Smeeth L, Miranda JJ. Low HDL cholesterol as a cardiovascular risk factor in rural, urban, and rural-urban migrants: PERU MIGRANT cohort study. Atherosclerosis. 2016; 246: 36-43.

5. Acharjee S, Boden WE, Hartigan PM, Teo KK, Maron DJ, Sedlis SP, Kostuk W, Spertus JA, Dada M, Chaitman BR. Low levels of high-density lipoprotein cholesterol and increased risk of cardiovascular events in stable ischemic heart disease patients: a post-hoc analysis from the COURAGE Trial (Clinical Outcomes Utilizing Revascularization and Aggressive Drug Evaluation). J Am Coll Cardiol. 2013; 62: 1826-33.

6. Schaefer EJ, Anthanont P, Diffenderfer MR, Polisecki E, Asztalos BF. Diagnosis and treatment of high density lipoprotein deficiency. Prog Cardiovasc Dis. 2016; 59: 97-106.

7. Pirillo A, Catapano AL. Pitavastatin and HDL: effects on plasma levels and function (s). Atheroscler Suppl. 2017.

8. World Health Organization. (2017). Cardiovascular diseases (CVDs). Updated May 2017.

9. Roth GA, Johnson C, Abajobir A, Abd-Allah F, Abera SF, Abyu G, Ahmed M, Aksut B, Alam T, Alam K, Alla F, Alvis-Guzman N, Amrock S, et al. Global, regional, and national burden of cardiovascular diseases for 10 causes, 1990 to 2015. J Am Coll Cardiol. 2017; 70: 1-25.

10. Adams V, Erbs S. Influence of exercise training on HDL function. Clin Lipidol. 2014; 9: 395-8.

11. Kodama S, Tanaka S, Saito K, Shu M, Sone Y, Onitake F, Suzuki E, Shimano H, Yamamoto S, Kondo K. Effect of aerobic exercise training on serum levels of high-density lipoprotein cholesterol: a meta-analysis. Arch Intern Med. 2007; 167: 999-1008.

12. Wang $\mathrm{Y}, \mathrm{Xu} \mathrm{D}$. Effects of aerobic exercise on lipids and lipoproteins. Lipids Health Dis. 2017; 16: 132.

13. World Health Organization. Global recommendations on physical activity for health. 2010.

14. Blazek A, Rutsky J, Osei K, Maiseyeu A, Rajagopalan S. Exercise-mediated changes in high-density lipoprotein: impact on form and function. Am Heart J. 2013; 166: 392-400.
15. Klancic T, Woodward L, Hofmann SM, Fisher EA. High density lipoprotein and metabolic disease: potential benefits of restoring its functional properties. Mol Metab. 2016; 5: 321-7.

16. Li YH, Ueng KC, Jeng JS, Charng MJ, Lin TH, Chien KL, Wang CY, Chao TH, Liu PY, Su CH, Chien SC, Liou CW, Tang SC, et al. 2017 Taiwan lipid guidelines for high risk patients. J Formos Med Assoc. 2017; 116: 217-48.

17. Ahmad WM, Aleng NA, Halim NA. Male and female differences in the High Density Lipoprotein (HDL) and progression of diabetic disease in coronary heart disease patients. Appl Math Sci. 2013; 7: 1825-38.

18. Greene NP, Martin SE, Crouse SF. Acute exercise and training alter blood lipid and lipoprotein profiles differently in overweight and obese men and women. Obesity. 2012; 20: 1618-27.

19. Hayashino Y, Jackson JL, Fukumori N, Nakamura F, Fukuhara S. Effects of supervised exercise on lipid profiles and blood pressure control in people with type 2 diabetes mellitus: a meta-analysis of randomized controlled trials. Diabetes Res Clin Pract. 2012; 98: 349-60.

20. Hur S, Kim SR. The effects of exercise therapy on CVD risk factors in women. J Phys Ther Sci. 2014; 26: 1367-70.

21. Durstine JL, Grandjean PW, Davis PG, Ferguson MA, Alderson NL, DuBose KD. Blood lipid and lipoprotein adaptations to exercise. Sports Med. 2001; 31: 1033-62.

22. Imamoglu O, Atan T, Kishali N, Burmaoglu G, Akyol P, Yildirim K. Comparison of lipid and lipoprotein values in men and women differing in training status. Biol Sport. 2005; 22: 261.

23. Brinkley TE, Halverstadt A, Phares DA, Ferrell RE, Prigeon RL, Hagberg JM, Goldberg AP. Hepatic lipase gene-514C> $\mathrm{T}$ variant is associated with exercise training-induced changes in VLDL and HDL by lipoprotein lipase. J Appl Physiol. 2011; 111: 1871-6.

24. Li Y, He PP, Zhang DW, Zheng XL, Cayabyab FS, Yin WD, Tang CK. Lipoprotein lipase: from gene to atherosclerosis. Atherosclerosis. 2014; 237: 597-608.

25. Kobayashi J, Mabuchi H. Lipoprotein lipase and atherosclerosis. Ann Clin Biochem. 2015; 52: 632-7.

26. Silva ER, Foster D, Harper MM, Seidman CE, Smith JD, Breslow JL, Brinton EA. Alcohol consumption raises HDL cholesterol levels by increasing the transport rate of apolipoproteins AI and A-II. Circulation. 2000; 102: 2347-52.

27. Brien SE, Ronksley PE, Turner BJ, Mukamal KJ, Ghali WA. Effect of alcohol consumption on biological markers associated with risk of coronary heart disease: systematic review and meta-analysis of interventional studies. BMJ. 2011; 342: d636.

28. He BM, Zhao SP, Peng ZY. Effects of cigarette smoking on HDL quantity and function: implications for atherosclerosis. J Cell Biochem. 2013; 114: 2431-6. 
29. Shamai L, Lurix E, Shen M, Novaro GM, Szomstein S, Rosenthal R, Hernandez AV, Asher CR. Association of body mass index and lipid profiles: evaluation of a broad spectrum of body mass index patients including the morbidly obese. Obes Surg. 2011; 21: 42-7.

30. Zamiriam M, Zibaeenezhad M, Mozaffari R. Correlation between HDL-C and smoking in teachers residing in Shiraz, Iran. Int Cardiovasc Res J. 2011; 5: 61-5.

31. Ruel I, Gaudet D, Perron P, Bergeron J, Julien P, Lamarche B. Effect of obesity on HDL and LDL particle sizes in carriers of the null P207L or defective D9N mutation in the lipoprotein lipase gene: the Quebec LipD Study. Int J Obes. 2003; 27 : 631-7.

32. Overview of Health Promotion in Taiwan. [cited 2016 09/08/2016]. From: http:/www.hpa.gov.tw/English/ file/ContentFile/201502030333474650/Health\%20 Promotion\%20in\%20Taiwan.pdf.

33. Prati D, Taioli E, Zanella A, Della Torre E, Butelli S, Del Vecchio E, Vianello L, Zanuso F, Mozzi F, Milani S. Updated definitions of healthy ranges for serum alanine aminotransferase levels. Ann Intern Med. 2002; 137: 1-10.
34. Wu WC, Wu CY, Wang YJ, Hung HH, Yang HI, Kao WY, $\mathrm{Su}$ CW, Wu JC, Chan WL, Lin HC. Updated thresholds for serum alanine aminotransferase level in a large-scale population study composed of 34346 subjects. Aliment Pharmacol Ther. 2012; 36: 560-8.

35. Siddiqui MS, Sterling RK, Luketic VA, Puri P, Stravitz RT, Bouneva I, Boyett S, Fuchs M, Sargeant C, Warnick GR. Association between high-normal levels of alanine aminotransferase and risk factors for atherogenesis. Gastroenterology. 2013; 145: 1271-9. e3.

36. Connelly MA, Shalaurova I, Otvos JD. High-density lipoprotein and inflammation in cardiovascular disease. Transl Res. 2016; 173: 7-18.

37. Yamada T, Hara K, Kadowaki T. Chewing betel quid and the risk of metabolic disease, cardiovascular disease, and all-cause mortality: a meta-analysis. PLoS One. 2013; 8: e70679.

38. Shafique K, Zafar M, Ahmed Z, Khan NA, Mughal MA, Imtiaz F. Areca nut chewing and metabolic syndrome: evidence of a harmful relationship. Nutr J. 2013; 12: 67. 